\title{
Combustión sin Llama de Mezclas Pobres Metano-Aire sobre Óxido de Magnesio Adicionado con Óxido de Calcio
}

\author{
Elías de J. Gómez, Mauricio E. Sánchez y Javier A. Jaramillo \\ Universidad de Antioquia, Departamento de Ingeniería Química, Grupo Ciencia y Tecnología del \\ Gas y Uso Racional de la Energía, Calle 67 No 53-108, bloque 18, oficina 435, Medellín-Colombia \\ (e-mail: egomez@udea.edu.co, msanchez@udea.edu.co, ajarami@udea.edu.co)
}

\begin{abstract}
Resumen
Se preparó material activo partiendo de óxido de magnesio $(\mathrm{MgO})$ grado industrial y grado reactivo adicionado con óxido de calcio $(\mathrm{CaO})$. En un lecho fijo empacado con partículas de material activo, se investigó el efecto que tienen la cantidad de $\mathrm{CaO}$ adicionado, la temperatura de calcinación, la velocidad espacial, el exceso de oxígeno y la composición de mezcla reactiva sobre la actividad, medida como conversión porcentual de metano. Se obtuvo combustión completa de metano para composiciones menores al límite inferior de inflamabilidad lo que permite evitar la emisión de $\mathrm{NO}_{x}$ producidos por mecanismo térmico. Las muestras preparadas con $\mathrm{MgO}$ grado industrial tuvieron actividad similar a las obtenidas con $\mathrm{MgO}$ grado reactivo adicionado con $\mathrm{CaO}$. Los materiales, de fácil preparación y bajo costo, mostraron alta estabilidad térmica antes y después de someterlos a reacción. Las propiedades de los sólidos preparados los hacen promisorios para aplicaciones en sistemas de combustión de gas natural y depuración de emisiones gaseosas.
\end{abstract}

\section{Flameless Combustion of Lean Methane-Air Mixtures over Magnesium Oxide with Addition of Calcium Oxide}

\begin{abstract}
Active material was prepared using industrial and reactive grade magnesium oxide ( $\mathrm{MgO})$ with the addition of calcium oxide $(\mathrm{CaO})$. In a fixed packed bed containing particles of active material, the effects of quantity of $\mathrm{CaO}$ added, calcination temperature, space velocity, oxygen excess and reactive mixture composition on activity, measured as percentage of methane conversion, were studied. Complete combustion was reached for compositions under the low limit of inflammability which allow avoiding the formation of thermal $\mathrm{NO}_{\mathrm{x}}$. Samples prepared with industrial grade $\mathrm{MgO}$ showed similar activity to those prepared with reactive grade $\mathrm{MgO}$ with the addition of $\mathrm{CaO}$. The materials, of low cost and easy preparation, showed high thermal stability before and after reaction tests. The properties of the prepared solids make them promising materials for natural gas combustion and gas emission treatment systems.
\end{abstract}

Keywords: flameless combustion, methane-air, fixed bed, calcium oxide, magnesium oxide 


\section{INTRODUCCIÓN}

La combustión térmica de hidrocarburos se caracteriza por la presencia de llama y altas temperaturas, con impactos negativos para el ambiente y la salud humana debido a las emisiones de hidrocarburos inquemados, óxidos de nitrógeno y monóxido de carbono. Entre las alternativas para solucionar los problemas mencionados, está el uso de superficies sólidas químicamente activas para la oxidación total de hidrocarburos, en mezclas de composición por debajo del límite inferior de inflamabilidad y a menor temperatura que la requerida en combustión térmica (Zwinkels et al., 1993)

Los materiales más activos para combustión sin llama de hidrocarburos gaseosos son los metales nobles y los óxidos simples de metales de transición (Requies et al., 2008; Yoshida et al., 2008; Gluhoi y Nieuwenhuys, 2007; Ramadj et al., 2007; Persson et al., 2007; Zhimin et al., 2007; Choudhary et al., 2002; Pfefferle y Pfefferle, 1987). Los metales nobles tienen alto costo, son inestables a altas temperaturas y de poca disponibilidad. Los óxidos de metales de transición, aunque menos costosos que los metales nobles, también presentan inestabilidad térmica y algunos tienen baja disponibilidad.

La necesidad de materiales activos, térmicamente estables y de bajo costo, ha llevado al estudio de óxidos mixtos (Petrovic et al., 2008; Chiarello et al., 2006; Campagnoli et al., 2005; Chen, et al., 2005), hexaaluminatos y óxidos de metales alcalinotérreos. Los dos últimos presentan alta estabilidad térmica pero actividad inferior a la de metales nobles y óxidos de metales de transición (Baylet et al., 2008; Ren et al, 2007; Li y Wang, 2007; Ersson et al., 2006; Choudhary et al, 2002). Los hexaaluminatos son un poco más activos que el óxido de magnesio, el más promisorio de los óxidos de metales alcalinotérreos, pero de menor estabilidad térmica y más difícil preparación (Berg y Järås, 1995).

El estudio de oxidación de metano sobre óxido de magnesio puro o dopado con otros elementos como litio, hierro, cobalto y calcio viene aumentando en interés (Teng, et al., 2007; Spretz et al., 2000; Berg y Järås, 1994; Aigler y Lunsford, 1991). El sistema litio-óxido de magnesio muestra mayor selectividad hacia etano y etileno, por acoplamiento oxidativo, que hacia dióxido de carbono por oxidación total (Dubois y Cameron, 1990; Ito et al., 1987). Cuando el MgO se dopa con cobalto o hierro se da la oxidación total del metano promovida por el metal de transición (Ulla et al., 2001; Spretz et al., 2000). Algunos estudios plantean que la presencia de calcio en la superficie del óxido de magnesio aumenta de manera considerable la actividad para oxidación parcial de metano (Aigler y Lunsford, 1991). Berg y Jarås (1994) encontraron que el óxido de magnesio puro, usado frecuentemente como soporte de metales nobles por su alta estabilidad térmica, tiene alguna actividad para la oxidación total de metano en exceso de oxígeno.

Las investigaciones sobre oxidación de metano con óxido de magnesio se hicieron con reactivos de alta pureza y diferentes métodos de preparación, pero no se hallaron reportes que indiquen la obtención de material activo para combustión de metano, mediante el uso de ingredientes grado industrial y preparado mediante un proceso de bajo costo.

En este trabajo se investigó la combustión sin llama de mezclas pobres metano-aire, en lecho fijo empacado con partículas de material activo obtenido por secado y calcinación del hidróxido de magnesio. Las suspensiones acuosas de hidróxido se prepararon a partir de óxido de magnesio grado industrial (conteniendo impurezas de calcio) y con óxidos de magnesio y de calcio grado reactivo. Se investigó el efecto de la relación oxígeno a metano, velocidad espacial, temperatura de calcinación y contenido de calcio sobre la actividad y estabilidad térmica.

Se desarrolló un material activo y estable, de fácil preparación, con materias primas de alta disponibilidad y bajo costo, promisorio para aplicaciones en sistemas de combustión de gas natural y depuración de emisiones gaseosas a escala industrial. Las características de actividad del óxido de magnesio preparado promueven la combustión de metano sin emisiones detectables de CO e hidrocarburos. Su alta actividad permite la combustión de mezclas metano aire cuya composición esté abajo del límite inferior de inflamabilidad para que la temperatura de reacción no supere $1500^{\circ} \mathrm{C}$, evitando de esta manera las emisiones de $\mathrm{NO}_{\mathrm{x}}$ térmicos. 


\section{MATERIALES Y MÉTODOS}

\section{Preparación del material activo}

Las fuentes para obtener el material activo fueron: a) óxido de magnesio industrial $(95.00 \% \mathrm{MgO}$; $0.90 \% \mathrm{CaO} ; 1.20 \% \mathrm{SiO}_{2} ; 0.50 \% \mathrm{Fe}_{2} \mathrm{O}_{3} ; 0.50 \% \mathrm{Al}_{2} \mathrm{O}_{3}$ y $1.84 \%$ inertes) y b) mezclas de óxido de magnesio alta pureza (Aldrich, $\mathrm{MgO}>99.80 \%$ en peso y $\mathrm{CaO}<0.02 \%$ ) y óxido de calcio alta pureza (Aldrich, $\mathrm{CaO}>99.80 \%$ y $\mathrm{MgO}<0.02 \%$ ) al $0.00,0.25,0.50$ y $1.00 \%$ en peso. Se prepararon suspensiones acuosas de una parte de sólido por cuatro de agua, con agitación mecánica durante 1.50 horas; se separó el sedimento después de 12 horas y se secó en estufa a $125^{\circ} \mathrm{C}$ por 36 horas. El sólido seco se compactó en una matriz de acero, se trituró y tamizó para conformar partículas con tamaños entre 2.00 y $2.36 \mathrm{~mm}$ que luego se calcinaron en aire durante 4 horas a 900, 1000 y $1100^{\circ} \mathrm{C}$.

\section{Instalación experimental}

La Fig. 1 muestra un esquema general de la instalación experimental. Se uso aire atmosférico y metano de alta pureza suministrado por AGA FANO. Para regular el flujo de mezcla reactiva metanoaire se fijó el caudal de metano y se ubicó, después del mezclador, un regulador de flujo másico total tipo hilo caliente dotado con interfaz de usuario y electrónica local, con una exactitud de \pm 0.01 litros estándar por minuto, SLPM. La concentración de metano en la mezcla alimentada al reactor se midió con un analizador de gases tipo infrarrojo no dispersivo (NDIR). El calentamiento y control de temperatura se realizó mediante una resistencia eléctrica manejada con un control de temperatura multicanal con precisión de $0.1^{\circ} \mathrm{C}$, dotado con algoritmo PID para el controlador (TIC) y termopares tipo R (TT). El reactor se construyó en material cerámico silico-aluminoso de $1.50 \mathrm{~cm}$ de diámetro interno, $1.20 \mathrm{~cm}$ de espesor de pared y $25 \mathrm{~cm}$ de longitud. Al reactor se acoplaron una resistencia eléctrica de $2 \mathrm{~kW}$ enrollada alrededor del tubo cerámico y cuatro termopares distribuidos uniformemente en dirección axial; el sistema se aisló con ladrillo refractario y manta cerámica. La mezcla reactiva y los productos de combustión se analizaron, después de pasar por una unidad de succión, acondicionamiento térmico y eliminación de humedad, con el analizador de gases NDIR adecuado para la medición simultanea de $\mathrm{CO}, \mathrm{CO}_{2}, \mathrm{CH}_{4}$ y $\mathrm{O}_{2}$. Debido a la posibilidad de controlar la temperatura máxima de reacción sin que sobrepase $1400^{\circ} \mathrm{C}$, no se midieron las emisiones de NOx porque el mecanismo térmico de formación comienza a tener importancia por encima de $1500^{\circ} \mathrm{C}$.

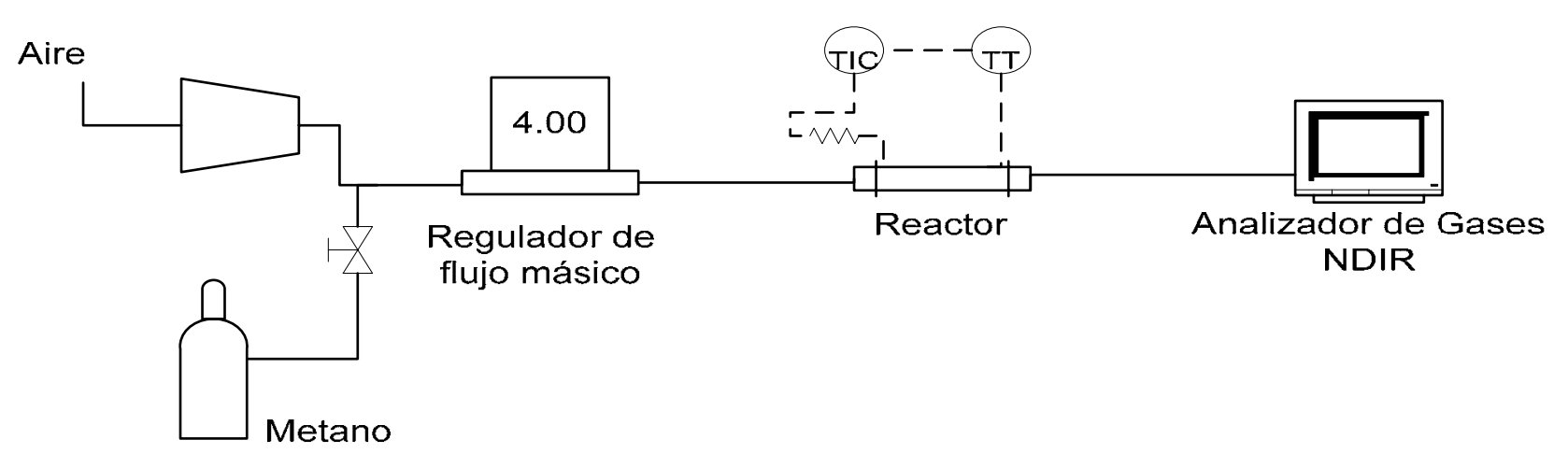

Fig. 1: Esquema general de la instalación experimental usada.

Pruebas de actividad

Se realizaron pruebas para investigar el efecto de: adición de óxido de calcio $(0.00,0.25,0.50$ y $1.00 \%$ en peso); temperatura de calcinación $\left(900,1000\right.$ y $\left.1100^{\circ} \mathrm{C}\right)$; velocidad espacial $(23500$, $83000,120000,154000$ y $180000 \mathrm{~h}^{-1}$ ) y exceso de oxígeno (mezclas aire-metano preparadas con $9.50,5.00,2.50$ y $1.25 \%$ de metano en volumen). En cada prueba se midieron las temperaturas necesarias para obtener conversiones de metano de 10, 20, 50, 70, 90 y 100\%. La velocidad espacial corresponde a la relación entre el flujo volumétrico de la mezcla reactiva alimentada en 
litros/hora y el volumen del reactor en litros. La conversión de metano se calcula como la relación porcentual entre las moles de metano que reaccionan y las moles de metano alimentadas al reactor.

\section{Pruebas de estabilidad}

La estabilidad del material a altas temperaturas y en condiciones de reacción, se investigó por los cambios presentados en superficie específica y cristalinidad. El área superficial B.E.T. del material calcinado, antes y después de usarlo en la reacción de combustión, se determinó por adsorción de nitrógeno a 77K en un equipo marca QUANTA CHROME, modelo Nova-1000. Previo a la medida del área todas las muestras se evacuaron por $3 \mathrm{~h}$ a $300^{\circ} \mathrm{C}$, para eliminar impurezas adsorbidas y humedad. Se hicieron análisis por Difracción de Rayos $X$ de polvos utilizando un equipo marca BRUKER, modelo D8 Advance, filtro de Fe, K- $\alpha$ del Co $(1.78897 \mathrm{~nm})$ dotado con detector PSD; los ensayos se corrieron entre $30-80^{\circ}$ utilizando un paso de $0.037^{\circ}$ y un tiempo en cada punto de 0.50 segundos. Los análisis se hicieron a las muestras de sólido calcinado, antes y después de la reacción de combustión.

\section{RESULTADOS Y DISCUSIÓN}

\section{Efecto de la adición de óxido de calcio}

La Fig. 2 muestra las curvas de conversión de metano en función de la temperatura, para la combustión de mezcla al $2.50 \%$ de metano en volumen, sobre material activo obtenido de óxido de magnesio de alta pureza adicionado con $0.25,0.50,0.75$ y $1.00 \%$ en peso de óxido de calcio, calcinadas a $1000^{\circ} \mathrm{C}$. La velocidad espacial de la mezcla reactiva fue $83000 \mathrm{~h}^{-1}$.

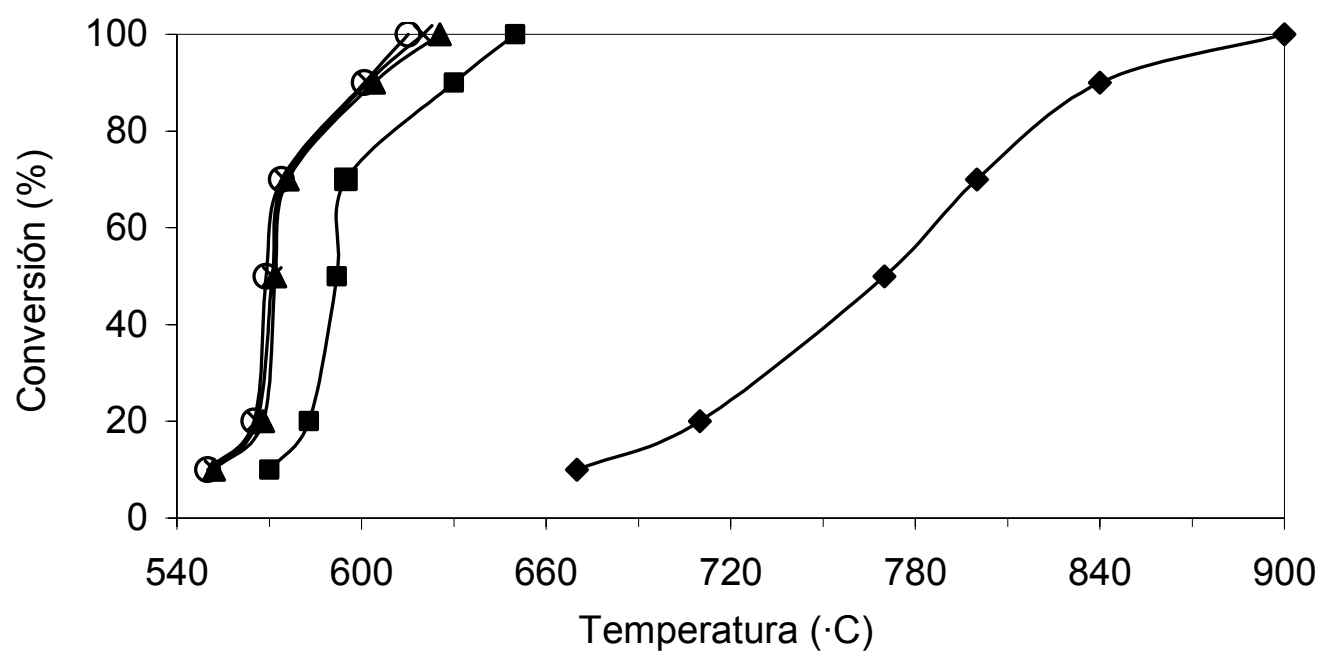

Fig. 2: Efecto del $\mathrm{CaO}$ sobre la actividad del MgO en combustión de metano: $(\diamond)$ 0.00\% CaO;

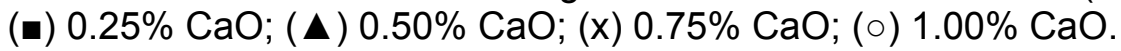

Es notable el efecto del óxido de calcio sobre la actividad. Con $0.25 \%$ de $\mathrm{CaO}$ adicionado, se requirieron $600^{\circ} \mathrm{C}$ para obtener $50 \%$ de conversión, mientras que para el MgO puro, igual conversión se logra a $780^{\circ} \mathrm{C}$. La diferencia es más acentuada a medida que aumenta la actividad, observándose que la conversión de $100 \%$ se tiene a $900^{\circ} \mathrm{C}$ para el sistema sin adición y $650^{\circ} \mathrm{C}$ para $0.25 \%$ adicionado de $\mathrm{CaO}$. A partir de $0.25 \%$ de $\mathrm{CaO}$, su incidencia sobre la actividad se va reduciendo, de tal manera que para conversión completa la diferencia es solo de $24.5^{\circ} \mathrm{C}$ entre adiciones de 0.25 y $0.50 \%$; dándose un incremento en la actividad aproximadamente 10 veces mayor entre $0.00 \%$ y $0.25 \%$ que entre 0.25 y $0.50 \%$. No hay grandes diferencias en cuanto al aumento en conversión para adiciones de $\mathrm{CaO}$ entre $0.50 \%$ y $1.00 \%$, indicando que cantidades de $\mathrm{CaO}$ superiores a $0.50 \%$ no son relevantes para mejorar la actividad del óxido de magnesio de manera considerable. El aumento en actividad se puede deber a la mayor basicidad superficial conferida por el $\mathrm{CaO}$, el cual puede migrar hacia la superficie durante la calcinación, tal como lo plantean Aigler y Lunsford (1991), 
Cunningham et al. (1988) y McCune y Wynblatt (1983). El analizador NDIR no mostró la presencia de $\mathrm{CO}$ ni en estas ni en ninguna otra de las pruebas de actividad.

Por tal razón en adelante se trabajó con óxido de magnesio industrial el cual contiene $0.90 \%$ de $\mathrm{CaO}$ y no requiere tratamientos especiales ni adición externa de dicho componente, lo que permite obtener materiales de fácil preparación y bajo costo.

\section{Efecto de la temperatura de calcinación}

En la Fig. 3 se observa la variación de la conversión de metano respecto a la temperatura, para combustión de mezcla al $2.5 \%$ de metano en volumen, sobre material activo proveniente de óxido grado industrial, calcinado a $900^{\circ} \mathrm{C}, 1000^{\circ} \mathrm{C}$ y $1100^{\circ} \mathrm{C}$, con velocidad espacial $83000 \mathrm{~h}^{-1}$. La actividad decrece a medida que se aumenta la temperatura de calcinación debido, posiblemente, a reducción del área superficial. La disminución en la actividad para el óxido calcinado a $1000^{\circ} \mathrm{C}$ respecto al calcinado a $900^{\circ} \mathrm{C}$, fue aproximadamente la misma que se dió para calcinación a $1100^{\circ} \mathrm{C}$ con respecto a $1000^{\circ} \mathrm{C}$. En general se observa que para conversiones arriba de $70 \%$, se requiere aumentar la temperatura de reacción en aproximadamente $15^{\circ} \mathrm{C}$ por cada incremento de $100^{\circ} \mathrm{C}$ en la temperatura de calcinación, para tener la misma conversión.

La actividad fue superior a la obtenida por Berg y Jaras (1994), quienes trabajaron con óxido de magnesio de alta pureza. Estos investigadores requirieron $875^{\circ} \mathrm{C}$ para tener $100 \%$ de conversión con calcinación a $900^{\circ} \mathrm{C}$, y $920^{\circ} \mathrm{C}$ cuando calcinaron a $1100^{\circ} \mathrm{C}$. La mejora en actividad del material obtenido a partir de ingredientes grado industrial, probablemente está relacionada con el calcio presente como impureza en el óxido a partir del cual se preparó la suspensión acuosa.

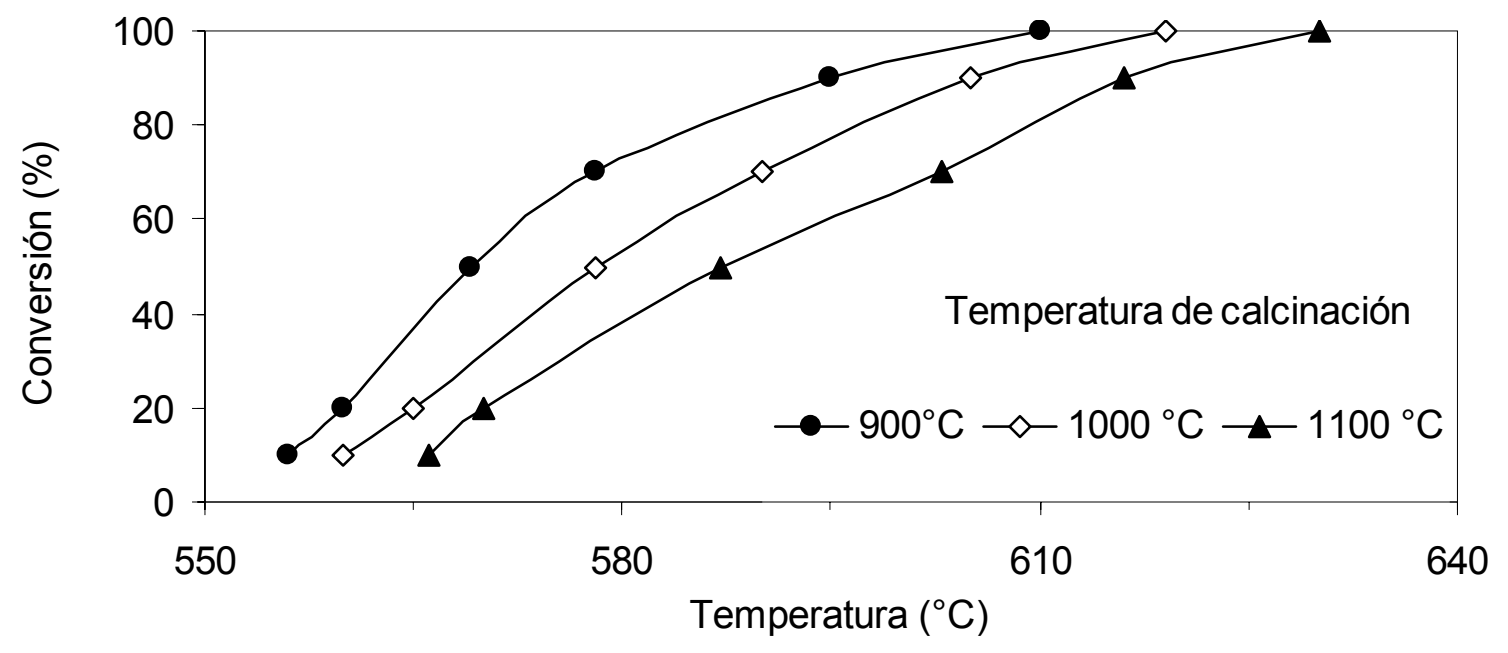

Fig. 3: Efecto de la temperatura de calcinación sobre la conversión de metano.

\section{Efecto de la velocidad espacial}

La Fig. 4 muestra la conversión en función de la temperatura, para combustión de mezcla al $2.5 \%$ en volumen de metano, sobre material preparado a partir de óxido de magnesio industrial, calcinado a $1000^{\circ} \mathrm{C}$, velocidades espaciales, GHSV, 23500, 83000, 120000,154000 y $180000 \mathrm{~h}^{-1}$.

A velocidad espacial de $23500 \mathrm{~h}^{-1}$, se requirieron $120^{\circ} \mathrm{C}$ menos que cuando la velocidad espacial fue $180000 \mathrm{~h}^{-1}$ para que la mezcla alcanzara $100 \%$ de conversión de metano. Las curvas muestran que para obtener altas conversiones de metano (superiores a $70 \%$ ), es necesario aumentar la temperatura de reacción en promedio $30^{\circ} \mathrm{C}$ por cada aumento de $30000 \mathrm{~h}^{-1}$ en la velocidad espacial. Teng et al. (2007) requirieron $830^{\circ} \mathrm{C}$ para obtener $100 \%$ de conversión de metano sobre $\mathrm{MgO}$ preparado por precipitación de carbonato y mezclas con relación $\mathrm{O}_{2} / \mathrm{CH}_{4}$ de 20 y velocidad espacial de $100000 \mathrm{~h}^{-1}$. Berg y Järas (1994) reportan, para velocidades espaciales de $100000 \mathrm{~h}^{-1}$ y relaciones 
$\mathrm{O}_{2} / \mathrm{CH}_{4}$ de 8 , la necesidad de alcanzar temperaturas del orden de $830^{\circ} \mathrm{C}$ para lograr una conversión de $83 \%$. En este trabajo solo se requirió una temperatura de $680^{\circ} \mathrm{C}$ para una velocidad de $120000 \mathrm{~h}^{-1}$ y relación $\mathrm{O}_{2} / \mathrm{CH}_{4}$ de 8 , mostrando de esta manera que el material aquí preparado tiene mayor actividad, lo que se traduce en menores tamaños de reactor y costos de material activo.

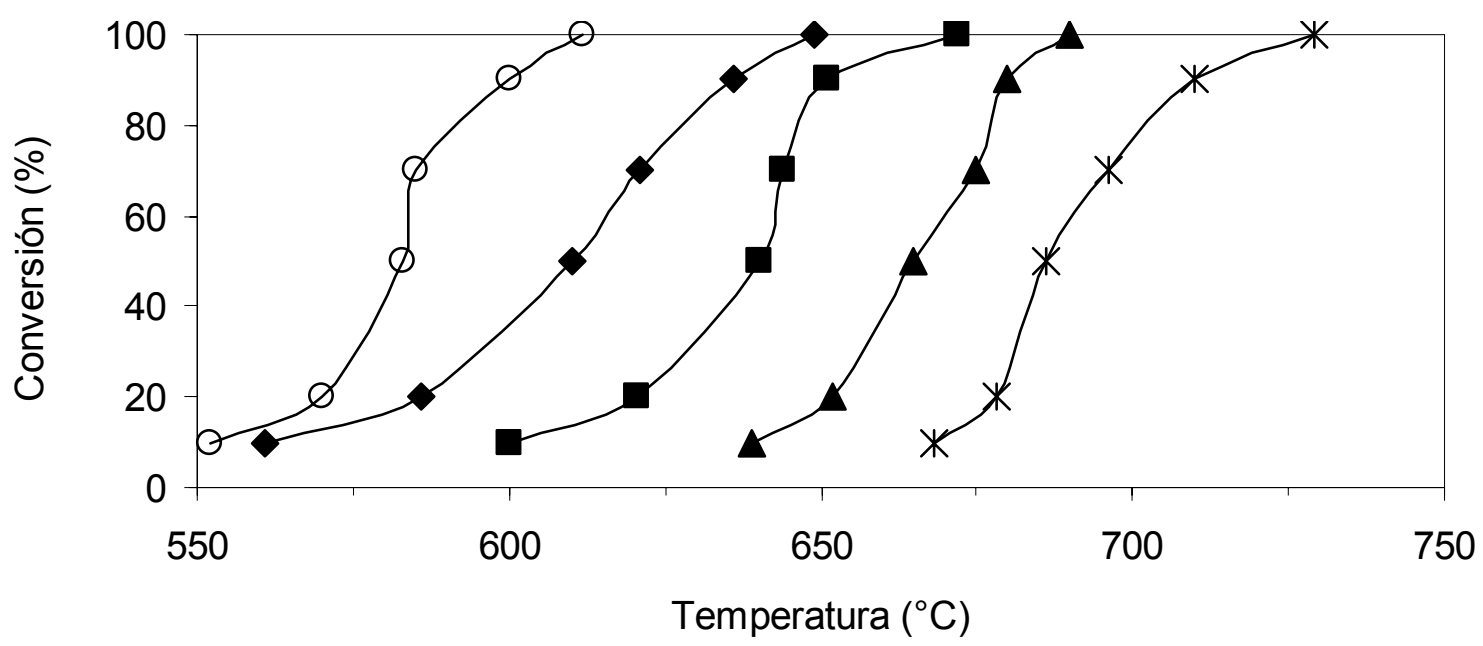

Fig. 4: Efecto de la velocidad espacial sobre la conversión de metano: (०) $23500 \mathrm{~h}^{-1}$; (•) $83000 \mathrm{~h}^{-1}$; (घ) $120000 \mathrm{~h}^{-1} ;$ ( $\left.\boldsymbol{\Delta}\right) 154000 \mathrm{~h}^{-1} ;(\mathbf{x}), 180000 \mathrm{~h}^{-1}$.

\section{Efecto del exceso de oxígeno}

En la Fig. 5 se presenta la dependencia de la conversión respecto a la temperatura, con material preparado a partir de óxido de magnesio industrial calcinado a $1000^{\circ} \mathrm{C}$ y concentraciones de metano $9.50,5.00,2.50$ y $1.25 \%$ en volumen, correspondientes respectivamente a la relación estequiométrica y a excesos de oxígeno aproximados de 100, 300 y $750 \%$. Las pruebas se realizaron para una velocidad espacial de $83000 \mathrm{~h}^{-1}$.

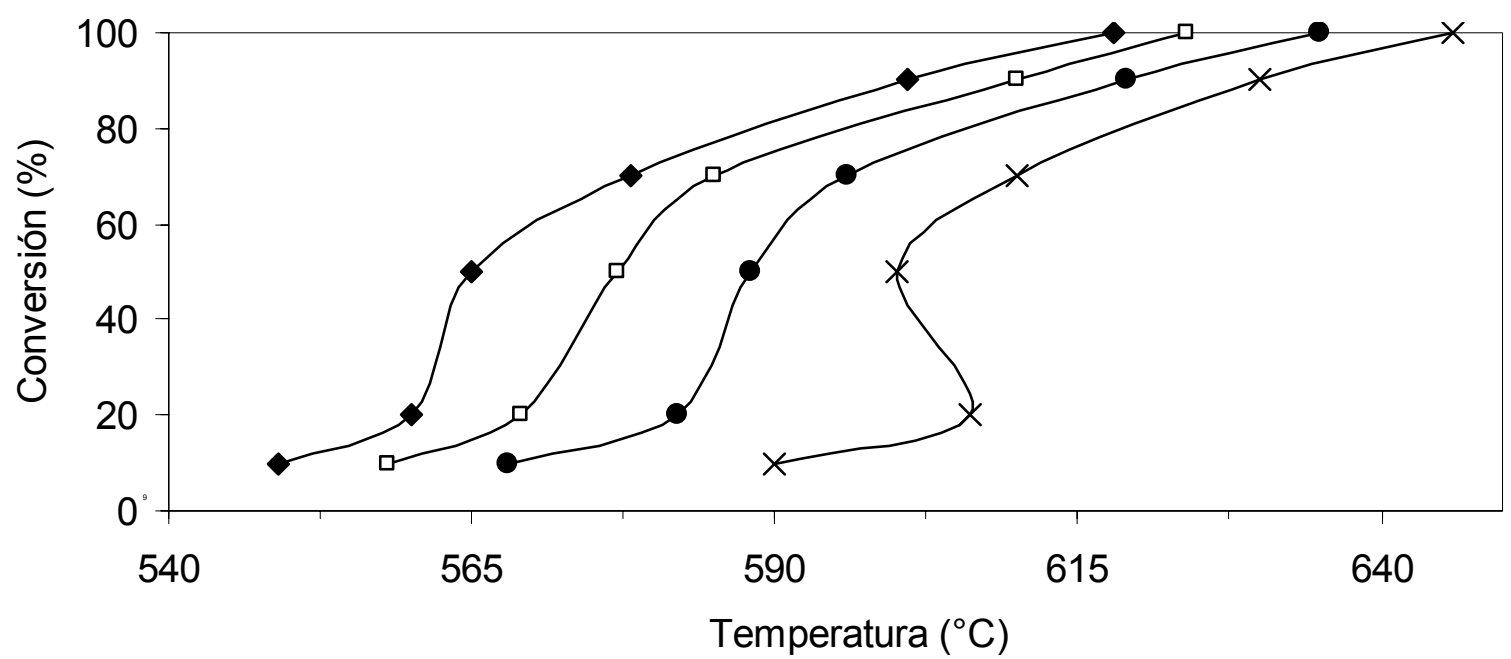

Fig. 5: Influencia del exceso de oxígeno sobre la conversión de metano: (匹) $1.25 \% \mathrm{CH}_{4}$; () $2.50 \%, \mathrm{CH}_{4} ;(\bullet) 5.00 \% \mathrm{CH}_{4} ;(\mathrm{x}) 9.50 \% \mathrm{CH}_{4}$.

Es notorio el efecto que tiene el oxígeno en exceso sobre la conversión completa de metano. Con el mayor exceso de oxígeno $(750 \%)$, se requirieron $615^{\circ} \mathrm{C}$ mientras que sin oxígeno en exceso (relación estequiométrica), la conversión total se logra con $645^{\circ} \mathrm{C}$. Para conversiones mayores a $70 \%$, si se duplica la relación molar oxígeno a metano, la temperatura requerida para tener la misma conversión 
disminuye alrededor de 10 a $12^{\circ} \mathrm{C}$. Esto indica que la disponibilidad de oxígeno en exceso en la corriente reactiva, mejora la actividad del óxido de magnesio para promover la combustión de mezclas metano-aire que se hallen por debajo del límite inferior de inflamabilidad. La misma tendencia ha sido reportada por otros investigadores (Berg y Jåras, 1994) quienes para una concentración de metano de $0.5 \%, 100000 \mathrm{~h}^{-1}$ y relaciones $\mathrm{O}_{2} / \mathrm{CH}_{4}$ de 4,8 y 16 encontraron conversiones de $58 \%, 83 \%$ y $90 \%$ respectivamente, a $830^{\circ} \mathrm{C}$.

Se esperaría que según las tendencias encontradas en este trabajo, al duplicar el exceso de oxígeno, la temperatura requerida para alcanzar la combustión completa se reduzca en promedio $10^{\circ} \mathrm{C}$ cada vez que se duplique la relación $\mathrm{O}_{2} / \mathrm{CH}_{4}$. Esto permite establecer que el material preparado a partir de $\mathrm{MgO}$ industrial presenta una actividad importante para la combustión de metano aun por debajo de los límites de inflamabilidad.

\section{Estabilidad térmica}

Difracción de rayos $X, D R X$ : Mediante el análisis de difracción de rayos $X$ realizado a materiales preparados con óxido de magnesio industrial, calcinados a $900^{\circ} \mathrm{C}, 1000^{\circ} \mathrm{C}$ y $1100^{\circ} \mathrm{C}$, y usados en combustión de metano, se analizó el posible cambio en cristalinidad. En la Tabla 1 puede apreciarse que no existe un desplazamiento significativo en la localización $(2 \theta)$ y de la intensidad relativa $\left(I / I_{0}\right)$ de los picos obtenidos respecto a los característicos de la estructura cristalina del MgO tipo periclasa, cambios que incluso podrían atribuirse por su orden de magnitud pequeño, a deformaciones mecánicas generadas durante la preparación de las muestras al momento de la realización de los análisis.

Tabla 1: Efecto de la temperatura de calcinación sobre la cristalinidad

\begin{tabular}{|c|c|c|c|c|c|c|}
\hline \multirow{3}{*}{ Materiales } & \multicolumn{6}{|c|}{ Temperatura de calcinación, ${ }^{\circ} \mathrm{C}$} \\
\cline { 2 - 7 } & \multicolumn{2}{|c|}{900} & \multicolumn{2}{c|}{1000} & \multicolumn{2}{c|}{1100} \\
\cline { 2 - 7 } & $2 \Theta$ & $\mathrm{I} / \mathrm{I}_{0}$ & $2 \Theta$ & $\mathrm{I} / \mathrm{I}_{0}$ & $2 \Theta$ & $\mathrm{I} / \mathrm{I}_{0}$ \\
\hline \multirow{2}{*}{$\begin{array}{c}\text { Grado } \\
\text { Industrial } \\
\text { usado }\end{array}$} & 43.07 & 100 & 43.07 & 100 & 43.07 & 100 \\
\cline { 2 - 7 } & 62.41 & 54 & 62.42 & 55 & 62.14 & 56 \\
\cline { 2 - 7 } & 78.76 & 16 & 78.73 & 16 & 78.73 & 16 \\
\hline
\end{tabular}

Los cambios en las intensidades del pico localizado en el ángulo $2 \Theta$ alrededor de $62.4^{\circ}$ son pequeños pero aun así puede notarse una tendencia de aumento a medida que la temperatura de calcinación se incrementa entre $900^{\circ} \mathrm{C}$ y $1100^{\circ} \mathrm{C}$. Este cambio puede atribuirse a la mayor cristalinidad de las muestras calcinadas a $1100^{\circ} \mathrm{C}$, dado que el material es prácticamente amorfo antes de calcinar y cristalino después de ser sometido a altas temperaturas. En adelante se decidió trabajar con muestras calcinadas a $1000^{\circ} \mathrm{C}$, por facilidad de fabricación y la poca incidencia observada a mayores temperaturas, para comparar el efecto de la naturaleza del material y el hecho de haber sido usado o no en la reacción de oxidación.

En la Tabla 2 puede observarse que para ambos materiales, a partir de $\mathrm{MgO}$ grado industrial y $\mathrm{MgO}$ grado reactivo, no hay diferencia apreciable en la ubicación de los picos característicos respecto a los de la periclasa. Solo se observaron unos pequeños incrementos en intensidad para las muestras antes y después de reacción, lo que puede deberse a que en el último caso la muestra permaneció mayor tiempo sometida a temperaturas altas, las cuales alcanzaron $1400^{\circ} \mathrm{C}$ en atmósfera oxidante.

Área superficial BET: En la Tabla 3 se presenta los valores de superficie específica de materiales preparados a partir de óxido de magnesio grado industrial y grado reactivo para muestras frescas y usadas a cada temperatura de calcinación. Los materiales frescos pierden área superficial a medida que aumenta la temperatura de calcinación, presentándose mayor porcentaje de reducción entre 1000 y $1100^{\circ} \mathrm{C}\left(15,5 \%\right.$ para el óxido de alta pureza y 15,8 para el comercial), que entre 900 y $1000^{\circ} \mathrm{C}$ (13,2 y $14,6 \%$ para alta pureza y comercial, respectivamente). 
Tabla 2: Efecto de la procedencia del material y el uso en reacción sobre la cristalinidad

\begin{tabular}{|c|c|c|c|c|}
\hline \multirow{2}{*}{ Material } & \multicolumn{2}{|c|}{ Antes de reacción } & \multicolumn{2}{c|}{ Después de reacción } \\
\cline { 2 - 5 } & $2 \Theta$ & $\mathrm{I} / \mathrm{I}_{0}$ & $2 \Theta$ & $\mathrm{I} / \mathrm{I}_{0}$ \\
\hline \multirow{3}{*}{ Grado industrial } & 43.16 & 100 & 43.07 & 100 \\
\cline { 2 - 5 } & 62.51 & 53 & 62.42 & 55 \\
\cline { 2 - 5 } & 78.82 & 14 & 78.73 & 16 \\
\hline \multirow{3}{*}{ Grado puro } & 43.13 & 100 & 43.07 & 100 \\
\cline { 2 - 5 } & 62.44 & 50 & 62.46 & 53 \\
\cline { 2 - 5 } & 78.76 & 14 & 78.74 & 13 \\
\hline
\end{tabular}

Tabla 3: Área superficial BET (en $\mathrm{m}^{2} \mathrm{~g}^{-1}$ ) de materiales preparados a partir de óxido de magnesio a diferentes condiciones.

\begin{tabular}{|c|c|c|c|c|}
\hline \multirow{2}{*}{$\begin{array}{c}\text { Temperatura } \\
\text { calcinación, } \\
{ }^{\circ} \mathrm{C}\end{array}$} & \multicolumn{2}{|c|}{$\begin{array}{c}\text { MgO } \\
\text { Grado reactivo }\end{array}$} & \multicolumn{2}{c|}{$\begin{array}{c}\text { MgO } \\
\text { Grado Industrial }\end{array}$} \\
\cline { 2 - 5 } & Fresco & Usado & Fresco & Usado \\
\hline 900 & 11.23 & 11.01 & 9.54 & 9.38 \\
\hline 1000 & 9.75 & 9.57 & 8.15 & 7.60 \\
\hline 1100 & 8.24 & 8.16 & 6.86 & 6.78 \\
\hline
\end{tabular}

No hay grandes diferencias en cuanto a variación de superficie específica entre los materiales obtenidos a partir de las dos fuentes investigadas (alta pureza y comercial). El comportamiento para el caso de materiales usados en reacción es bastante análogo. En todos los casos el área superficial hallada en este trabajo es menor que las reportadas por Berg y Järas (1995), quienes obtuvieron $43.4 \mathrm{~m}^{2} \mathrm{~g}^{-1}$ con calcinación $900^{\circ} \mathrm{C}$ y $24.2 \mathrm{~m}^{2} \mathrm{~g}^{-1}$ a $1100^{\circ} \mathrm{C}$; no obstante, el porcentaje de reducción del área $(44 \%)$ es bastante mayor que el presentado en este trabajo (26.2\% para óxido grado reactivo y $27.9 \%$ para óxido grado industrial).

La reacción de combustión de gas natural no causa una reducción considerable en el área superficial, con un promedio de $1.5 \%$ para los materiales provenientes de las dos fuentes (alta pureza y comercial). Si se compara este resultado con la superficie específica para calcinación a $1300^{\circ} \mathrm{C}$ obtenida por Berg y Järas (1995) $\left(9,2 \mathrm{~m}^{2} \mathrm{~g}^{-1}\right)$, considerando que el material estudiado en este trabajo estuvo sometido a temperaturas de hasta $1400^{\circ} \mathrm{C}$, se puede afirmar que el área superficial no se ve prácticamente alterada por las condiciones de reacción y cuando se somete a altas temperaturas la perdida de superficie específica es menor que la reportada por otros autores. Esto significa que el material preparado es estable térmicamente aun después de ser usado en combustión de gas natural a temperaturas altas.

\section{CONCLUSIONES}

Se prepararon materiales activos, para combustión sin llama de mezclas metano-aire, partiendo de óxido de magnesio grado industrial y grado reactivo por un método sencillo y de muy bajo costo. La actividad se observó altamente favorecida por la presencia de pequeñas cantidades de óxido de calcio, manifestándose este efecto de manera más notoria hasta una concentración de $0.50 \%$. No hubo diferencias significativas respecto a la actividad entre el material activo proveniente de óxido de magnesio grado reactivo con adición de calcio y el preparado con óxido de magnesio grado industrial con impurezas óxido de calcio. No se encontró un efecto considerable de la temperatura de 
calcinación sobre la actividad y la estabilidad térmica de los materiales, así la temperatura de reacción requerida para conversión completa de metano con calcinaciones a $1100^{\circ} \mathrm{C}$ fue solo $20^{\circ} \mathrm{C}$ mayor que para calcinaciones a $900^{\circ} \mathrm{C}$.

El aumento de la velocidad espacial del gas en un factor de 8 solo requirió $120^{\circ} \mathrm{C}$ adicionales en la temperatura de reacción alcanzando combustión completa a $730^{\circ} \mathrm{C}$, mostrando actividad aun para bajos tiempos de contacto superficie activa-gas. El material activo mostró capacidad de promover la combustión completa de las mezclas metano-aire para la composición estequiométrica y exceso de oxígeno; con $750 \%$ de exceso de oxígeno la temperatura de reacción estuvo $40^{\circ} \mathrm{C}$ por debajo de la requerida para relación estequiométrica.

Todos los materiales mostraron alta estabilidad térmica, evidenciada en la poca sensibilidad de la estructura cristalina y el área superficial a los cambios de temperatura y condiciones de operación, que en los casos más extremos alcanzaron los $1400^{\circ} \mathrm{C}$ y más de $72 \mathrm{~h}$ de reacción.

El hecho de poder controlar el rango de composiciones de mezcla alimentada, y con éste la temperatura máxima de los gases a $1400^{\circ} \mathrm{C}$, permite desarrollar un sistema de combustión de baja producción de $\mathrm{NO}_{x}$, sin presencia $\mathrm{CO}$, con un material activo estable, de fácil preparación, con materias primas de alta disponibilidad y bajo costo, que podría ser escalado para operación industrial limpia.

\section{AGRADECIMIENTOS}

A la Universidad de Antioquia (Comité para el Desarrollo de la Investigación, CODI) por la financiación. Al Grupo Ciencia y Tecnología del Gas y URE por el apoyo logístico.

\section{REFERENCIAS}

Aigler, J.M. y J.H. Lunnsford; Oxidative dimerization of methane over $\mathrm{MgO}$ and $\mathrm{Li}^{+} / \mathrm{MgO}$ monoliths, Appl. Catal.: 70(1), 29-42 (1991).

Baylet, A. y otros cuatro autores; High catalytic activity and stability of $P d$ doped hexaaluminate catalysts for the CH4 catalytic combustión, Appl. Catal. B: Environmental: 77(3-4), 237-247 (2008).

Berg, M. y S. Järas; Catalytic Combustion of Methane Over Magnesium Oxide, Appl. Catal. A. General: 114(2), 227-241 (1994).

Berg, M. y S. Järas; High temperature stable magnesium catalyst for catalytic combustion of methane: a comparison with manganese-substututed barium hexaaluminate, Catal. Today: 26(3-4), 223-229 (1995).

Campagnoli, E. y otros seis autores; Effect of preparation method on activity and stability of $\mathrm{LaMnO}_{3}$ and $\mathrm{LaCOO}_{3}$ catalysts for the flameless combustion of methane, Appl. Catal. B: Environmental: 55(2) 133-139 (2005).

Chen, M. y otros cuatro autores; Synthesis of nanoparticle Ce-Mg-O mixed oxide as efficient support for methane oxidation, J. Molec. Catal. A: Chemical: 237(1-2), 132-136 (2005).

Chiarello, G.L. y otros cuatro autores; Preparation by flame spray pyrolysis of $A B O_{3 \pm \delta}$ catalysts for the flameless combustion of methane, Catal. Today: 117(4), 549-553 (2006).

Choudhary, T.V., V.R. Banerjee y V.R. Choudhary; Catalyst for combustion of methane and lower alkanes, Appl. Catal. A. General: 234(1-2), 1-23 (2002).

Cunningham. J. y otros tres autores; Preparation and characterization of $\mathrm{MgO}$ powders having $\mathrm{Ca}^{2+}$ or $\mathrm{Ba}^{2+}$ as surface dopants, Catal. Today: 2(5), 557-567 (1988). 
Dubois J.L. y C.J. Cameron; Common features of oxidative coupling of methane cofeed catalyst, App. Catal. 67(1), 49-71 (1990).

Ersson, A., K. Persson, I.K. Adu y S.G. Järås; A comparison between hexaaluminates and perovskites for catalytic combustion applications, Catalysis Today: 112(1-4),157-160(2006).

Gluhoi, A.G. y B.E. Nieuwenhuys; Catalytic oxidation of saturated hydrocarbons on multicomponent $\mathrm{Au} / \mathrm{Al}_{2} \mathrm{O}_{3}$ catalysts: Effect of various promoters, Catalysis Today: 119(1-4), 305-310 (2007).

Ito, T., y otros tres autores; Oxidative dimerization of methane over Lithium-Promoted magnesium oxide catalyst, J. Am. Chem. Soc.: 107, 50-62 (1985).

Li, S. y X. Wang; Catalytic combustion of methane over Mn-substituted Ba-La-hexaaluminate nanoparticles, Journal of Alloys and Compounds: 432(1-2), 333-337(2007).

McCune R.C. y P. Wynblatt; Calcium Segregation to a Magnesium Oxide (100) Surface, J. Am. Ceram. Soc.: 66(2), 111-116 (1983).

Persson, K., K. Jansson y S.G. Järås; Characterisation and microstructure of Pd and bimetallic Pd-Pt catalysts during methane oxidation, J. Catal.: 245(2), 401-414 (2007).

Petrovic, S. y otros ocho autores; $\mathrm{LaMO}_{3}(\mathrm{M}=\mathrm{Mg}$, Ti, Fe) perovskite type oxides: Preparation, characterization and catalytic properties in methane deep oxidation, Appl. Catal. B: Environmental: 79(2), 186-198 (2008).

Pfefferle, L.D. y W.C. Pfefferle; Catalysis in combustion, Catal. Rev. Sci. Eng.: 29, 219-267 (1987).

Ramadj, O.M. y otros tres autores; Catalytic Combustion of Methane over High Copper-Loading ZSM5 Catalysts, J. Natural Gas Chemistry: 16(3), 258-265 (2007).

Ren, X. y otros tres autores; Catalytic properties of Fe and Mn modified lanthanum hexaaluminates for catalytic combustion of methane, Catal. Commun. (2007), doi:10.1016/j.catcom.2007.09.004.

Requies, J. y otros ocho autores; Palladium-manganese catalysts supported on monolith systems for methane combustión, Appl. Catal. B: Environmental: 79(2), 122-131 (2008).

Spretz, R. y otros tres autores; Fe/MgO formulations for the catalytic combustion of methane, J. Catal.: 194(2), 167-174 (2000).

Teng, F. y otros ocho autores; Effect of the flowing gases of steam and $\mathrm{CO}_{2}$ on the texture and catalytic activity for methane combustion of MgO powders: Micropor. Mesopor. Mater. doi:10.1016/j.micromeso.2007.07.013, (2007).

Ulla, M. A. y otros cuatro; Catalytic combustión of methane on Co/MgO: characterization of active cobalt sites, Appl. Catal. B: Environmenta: 29(3), 217-229 (2001).

Yoshida, H. y otros tres autores; Support effect on methane combustion over palladium catalysts, Appl. Catal. B: Environmental: 71(1-2), 70-79 (2007).

Zhimin, L. y otros cinco autores; Effect of $\mathrm{Fe}_{2} \mathrm{O}_{3}$ Loading Amount on Catalytic Properties of Monolithic $\mathrm{Fe}_{2} \mathrm{O}_{3} / \mathrm{Ce}_{0.67} \mathrm{Zr}_{0.33} \mathrm{O}_{2}-\mathrm{Al}_{2} \mathrm{O}_{3}$ Catalyst for Methane Combustión, J. of Rare Earths: 25(5), 585-589 (2007).

Zwinkels, M.F., S.G. Jaras y P. Menon; Catalytic Materials for High Temperature Combustion, Catal. Rev. Sci. Eng.: 35(3), 319-358 (1993). 